\title{
Solar Wind Turbulent Cascade from MHD to Sub-ion Scales: Large-size 3D Hybrid Particle-in-cell Simulations
}

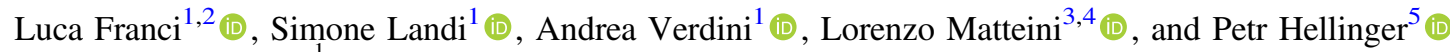 \\ ${ }^{1}$ Dipartimento di Fisica e Astronomia, Università di Firenze, Firenze, Italy \\ ${ }^{2}$ School of Physics and Astronomy, Queen Mary University of London, London, UK \\ ${ }^{3}$ Department of Physics, Imperial College London, UK \\ ${ }^{4}$ LESIA, Observatoire de Paris, Meudon, France \\ ${ }^{5}$ Astronomical Institute, CAS, Prague, Czech Republic \\ Received 2017 November 7; revised 2017 December 12; accepted 2017 December 18; published 2018 January 19
}

\begin{abstract}
Properties of the turbulent cascade from fluid to kinetic scales in collisionless plasmas are investigated by means of large-size 3D hybrid (fluid electrons, kinetic protons) particle-in-cell simulations. Initially isotropic Alfvénic fluctuations rapidly develop a strongly anisotropic turbulent cascade, mainly in the direction perpendicular to the ambient magnetic field. The omnidirectional magnetic field spectrum shows a double power-law behavior over almost two decades in wavenumber, with a Kolmogorov-like index at large scales, a spectral break around ion scales, and a steepening at sub-ion scales. Power laws are also observed in the spectra of the ion bulk velocity, density, and electric field, at both magnetohydrodynamic (MHD) and kinetic scales. Despite the complex structure, the omnidirectional spectra of all fields at ion and sub-ion scales are in remarkable quantitative agreement with those of a 2D simulation with similar physical parameters. This provides a partial, a posteriori validation of the 2D approximation at kinetic scales. Conversely, at MHD scales, the spectra of the density and of the velocity (and, consequently, of the electric field) exhibit differences between the 2D and 3D cases. Although they can be partly ascribed to the lower spatial resolution, the main reason is likely the larger importance of compressible effects in the full 3D geometry. Our findings are also in remarkable quantitative agreement with solar wind observations.
\end{abstract}

Key words: magnetohydrodynamics (MHD) - plasmas - solar wind - turbulence

\section{Introduction}

In situ data from solar and heliospheric spacecraft missions provide observations of the solar wind plasma and electromagnetic fluctuations in the frequency range $10^{-5} \mathrm{~Hz}<f<10^{2} \mathrm{~Hz}$. The power spectra of such fluctuations typically exhibit a power-law behavior over several decades in frequency, with different powerlaw indices at scales larger or smaller than about $1 \mathrm{~Hz}$, corresponding to the characteristic proton spatial scales (e.g., Alexandrova et al. 2009; Roberts 2010; Sahraoui et al. 2010; Chen 2016).

Measurements of the third-order structure functions verify that power-law spectra at scales well above the proton scales (hereafter MHD scales) result from a turbulent cascade (MacBride et al. 2005, 2008; Sorriso-Valvo et al. 2007). Recently, the exact law for the third-order structure function has been extended to the case of homogeneous incompressible Hall-MHD turbulence and applied to 2D HPIC simulations (Hellinger et al. 2017b). Those numerical results suggest that the cascade likely continues all the way down to sub-proton scales (hereafter kinetic scales) via the Hall term. Although direct evidence is still missing, this is further supported by the fact that the measured cascade rate in the solar wind is consistent with the proton heating rate (Vasquez et al. 2007; Marino et al. 2008; Stawarz et al. 2009). In this context, direct numerical simulations of turbulent plasmas not only are useful for interpreting the nature of the solar wind fluctuations at MHD scales but also represent a tool to understand how energy is channeled to protons and electrons.

At MHD scales, the solar wind fluctuations are predominantly Alfvénic: the magnetic field and the ion bulk velocity are observed to be dominated by their transverse components with respect to the ambient magnetic field. The former typically shows a Kolmogorov-like spectrum, with a slope of $\sim-5 / 3$, while the latter is usually flatter, with a spectral index closer to $-3 / 2$ (e.g., Podesta et al. 2007; Salem et al. 2009; Tessein et al. 2009; Wicks et al. 2011). The electric field is strongly coupled to the ion bulk velocity (Chen et al. 2011).

When reaching the proton kinetic scales, both the magnetic and velocity spectra are observed to steepen. The former has a spectral index varying between -4 and -2 at sub-ion scales (Leamon et al. 1998; Smith et al. 2006; Sahraoui et al. 2010 e.g.,), but typically close to $\sim-2.8$ in the range between the ion and the electron scales (Alexandrova et al. 2009, 2012; Sahraoui et al. 2013). The latter decouples from the magnetic field and typically shows a steeper and much more variable power law (e.g., Śafránková et al. 2016). On the contrary, the electric field spectrum flattens (Bale et al. 2005; Sahraoui et al. 2009; Salem et al. 2012), dominating over the magnetic field's, with a typical spectral index of about -0.8 (e.g., Stawarz et al. 2016; Matteini et al. 2017), as predicted from the generalized Ohm's law (Franci et al. 2015a). Moreover, an increase in the magnetic compressibility (Salem et al. 2012; Kiyani et al. 2013) and a reduced variance anisotropy (Podesta \& TenBarge 2012) are also observed.

Finally, the spectrum of the density fluctuations is very peculiar. It exhibits, unlike all the other fields, a sort of triplepower-law behavior, with consequently two different breaks (Chandran et al. 2009; Šafránková et al. 2015). Its slope is compatible with $\sim-5 / 3$ in the MHD range, close to -1 and $\sim-2.8$ at scales slightly larger or smaller than the ion characteristic scales, respectively (Chen et al. 2012).

Determining the physical scale(s) and process(es) associated with the ion-scale break in the magnetic field spectrum is not straightforward. The ion inertial length, $d_{\mathrm{i}}$, and the ion 
gyroradius, $\rho_{\mathrm{i}}=d_{\mathrm{i}} \sqrt{\beta}$ ( $\beta$ being the ion plasma beta), are very similar under typical solar wind conditions $(\beta \sim 1)$. Observational results suggest that the transition likely occurs in correspondence with the larger of the two when they are well separated (Chen et al. 2014) or a combination of the two in the intermediate-beta regime (Bruno \& Trenchi 2014). Although the nature of the power-law behavior of the solar wind fluctuations at kinetic scales is still under debate, an increasing consensus has recently emerged about the fundamental role of coherent structures and magnetic reconnection in shaping the magnetic field spectrum near and below the ion scales (e.g., Franci et al. 2016, 2017b; Cerri \& Califano 2017; Loureiro \& Boldyrev 2017; Mallet et al. 2017).

In the past decade, many 2D numerical simulations near and below the ion scales were able to reproduce some aspects of plasma turbulence (e.g., Gary et al. 2008; Parashar et al. 2009; Markovskii \& Vasquez 2010; Camporeale \& Burgess 2011; Servidio et al. 2012; Wan et al. 2012; Cerri et al. 2016; Cerri \& Califano 2017). Despite the different large-scale initial conditions or forcing, a certain agreement is observed between their results, suggesting that the spectral behavior at kinetic scales is quite independent from the dynamics at MHD scales.

Recently, very high resolution 2D HPIC simulations fully covered the transition between the fluid and the kinetic scales. In particular, Franci et al. (2015a, 2015b) produced extended turbulent spectra with well-defined power laws for the magnetic, ion bulk velocity, density, and electric fluctuations, in agreement with solar wind observations. Moreover, Franci et al. (2016) unambiguously determined the ion-scale break in the magnetic field spectrum and recovered the observed dependence on the ion characteristic scales.

Although 2D HPIC simulations allow for simultaneously covering three decades in wavenumber across the ion scales, they imply limitations for the turbulent dynamics and for the onset of plasma instabilities. This is particularly relevant for the solar wind plasma, in which the spherical expansion of the mean flow on the one hand shapes the turbulent anisotropy at MHD scale (Dong et al. 2014; Verdini \& Grappin 2015, 2016) and on the other hand continuously drives instabilities of proton velocity distribution functions at kinetic scales (Hellinger et al. 2015, 2017a).

In recent years, an increasing number of 3D numerical simulations have investigated the development and the properties of the turbulent cascade around and below the ion scales, employing different methods and models (e.g., Shaikh \& Shukla 2009; Chang et al. 2011; Howes et al. 2011; Gary et al. 2012; Boldyrev et al. 2013; Gómez et al. 2013; Meyrand \& Galtier 2013; Rodriguez Imazio et al. 2013; TenBarge \& Howes 2013; Passot et al. 2014; Servidio et al. 2015; Vasquez 2015; Wan et al. 2015, 2016; Cerri et al. 2017; Valentini et al. 2017). However, to our knowledge, 3D kinetic simulations have not been accurate enough yet to obtain clear and extended power laws for the electromagnetic and plasma fluctuations, spanning both the MHD and the kinetic range, consistently with solar wind observations. Moreover, while the different behavior of intermittency and dissipation between 2D and 3D has been investigated (Wan et al. 2016), a quantitative comparison of the spectral properties for all fields (e.g., spectral indices, scale of the break) in the two cases is still lacking in the literature.

In this work, we extend our 2D numerical studies, investigating the physics behind the transition from MHD to kinetic scales in the full 3D geometry, which allows for a more realistic representation of the solar wind turbulent plasma. Via a quantitative analysis of the spectral properties, we test the limitations of the reduced geometry and validate the results of our previous 2D HPIC simulations.

The paper is organized as follows. In Section 2, we introduce the numerical and physical setup, along with the definitions of spectra. In Section 3, we describe our numerical results, first focusing on the development of the turbulent cascade (Section 3.1) and later on the spectral properties of the quasistationary fully developed turbulent state (Section 3.2). In Section 3.3, we provide a direct quantitative comparison of our 3D results with those of a previous 2D simulation of comparable size and similar physical parameters. Finally, in Section 4, we summarize and discuss our findings.

\section{Numerical Setup and Initial Conditions}

We employ the hybrid particle-in-cell code CAMELIA (Current Advance Method Et cycLIc leApfrog), where the electrons are considered as a massless, charge-neutralizing fluid, whereas the ions (protons) are described by a particle-incell model and are advanced by the Boris scheme (see Matthews 1994; Franci et al. 2017a, for details on the numerical scheme and code performance).

The characteristic spatial and temporal units in this model are the ion inertial length $d_{\mathrm{i}}=v_{A} / \Omega_{\mathrm{i}}$, where $v_{A}$ is the Alfvén speed and $\Omega_{\mathrm{i}}^{-1}$ is the inverse ion gyrofrequency.

We ran a simulation employing a periodic cubic grid with spatial resolution $0.25 d_{\mathrm{i}}, 512^{3}$ grid points, box size $L_{\mathrm{box}}=$ $128 d_{\mathrm{i}}$, and 2048 particle-per-cell (ppc) representing protons. The resistive coefficient is set to the value $\eta=$ $1.5 \times 10^{-3} 4 \pi v_{A} c^{-1} \Omega_{\mathrm{i}}^{-1}$, to prevent the accumulation of magnetic energy at the smallest scales. The ions are advanced with a time step $\Delta t=0.05 \Omega_{\mathrm{i}}^{-1}$, while the magnetic field $\boldsymbol{B}$ is advanced with a smaller time step, $\Delta t_{B}=\Delta t / 10$.

We assume a uniform magnetic field directed along the $z$ direction, $\boldsymbol{B}_{0}=B_{0} \hat{z}=1$. Fields and wavevectors are always defined as parallel $(\|)$ and perpendicular $(\perp)$ with respect to $\boldsymbol{B}_{0}$. We also assume a uniform density, equal for ions and electrons, $n_{\mathrm{i}}=n_{\mathrm{e}}=n$. Both species have uniform and isotropic temperatures, $T_{\mathrm{i}}$ and $T_{\mathrm{e}}$, such that $\beta_{\mathrm{i}}=\beta_{\mathrm{e}}=0.5$ (where $\beta_{\mathrm{i}, \mathrm{e}}=8 \pi n K_{\mathrm{B}} T_{\mathrm{i}, \mathrm{e}} / B_{0}^{2}$ represents the plasma betas and $K_{\mathrm{B}}$ is the Boltzmann's constant).

We initialize the simulation by imposing linearly polarized shear Alfvénic fluctuations with random phases, i.e., the fluctuations are perpendicular to the plane defined by their wavevector and the mean field. In this way, the initial kinetic and magnetic fluctuations are almost at equipartition (within $10 \%$ ) and have vanishing correlation. The velocity fluctuations are divergenceless, and the density fluctuations are vanishing (in the limit of numerical noise). Fourier modes of equal amplitude are excited in the range $k_{0}<k<k_{\text {inj }}$, where $k=$ $\sqrt{k_{x}^{2}+k_{y}^{2}+k_{z}^{2}}$. The minimum wavenumber is $k_{0}=2 \pi /$ $L_{\text {box }} \simeq 0.05 d_{\mathrm{i}}^{-1}$, while the maximum injection scale is $k_{\mathrm{inj}} \simeq 0.25 d_{\mathrm{i}}^{-1}$.

We define the 3D axisymmetric spectrum of a generic field $\Psi$ as the energy of its 3D Fourier modes averaged over rings delimited by $k_{\perp}$ and $k_{\perp}+d k_{\perp}$ (where $k_{\perp}$ is the perpendicular wavenumber and $d k_{\perp}=k_{0}$ ),

$$
P_{3 \mathrm{D}}\left(k_{\perp}, k_{\|}\right)=\frac{1}{k_{\perp}} \sum_{\sqrt{k_{x}^{2}+k_{y}^{2}}=k_{\perp}} \hat{\Psi}_{3 \mathrm{D}}^{2}\left(k_{x}, k_{y}, k_{z}\right),
$$




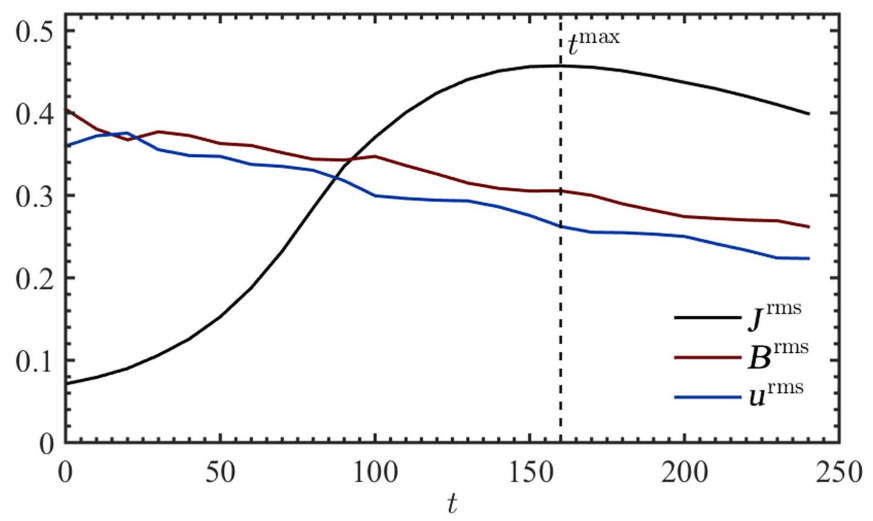

Figure 1. Time evolution of the rms of the current density, $\boldsymbol{J}$ (black line), of the magnetic field, $\boldsymbol{B}$ (red), and ion bulk velocity, $\boldsymbol{u}$ (blue). The dashed vertical line marks the time of the maximum of $J^{\mathrm{rms}}, t_{\max }=160 \Omega_{\mathrm{i}}^{-1}$.

where $\hat{\Psi}$ are $\Psi$ 's Fourier coefficients. This is statistically representative of a random $2 \mathrm{D}$ slice of the $3 \mathrm{D}$ Fourier space in the direction of $\boldsymbol{k}_{\|}$.

The 2D spectrum is obtained by integrating the energy of the Fourier modes contained in the above-defined rings,

$$
\begin{aligned}
P_{2 \mathrm{D}}\left(k_{\perp}, k_{\|}\right) & =\sum_{\sqrt{k_{x}^{2}+k_{y}^{2}}=k_{\perp}} \hat{\Psi}_{3 \mathrm{D}}^{2}\left(k_{x}, k_{y}, k_{z}\right) \\
& =k_{\perp} P_{3 \mathrm{D}}\left(k_{\perp}, k_{\|}\right) .
\end{aligned}
$$

A further integration yields the 1D reduced perpendicular and parallel spectra,

$$
\begin{gathered}
P_{1 \mathrm{D}, \perp}\left(k_{\perp}\right)=\sum_{k_{\|}} P_{2 \mathrm{D}}^{\Psi}\left(k_{\perp}, k_{\|}\right), \\
P_{1 \mathrm{D}, \|}\left(k_{\|}\right)=\sum_{k_{\perp}} P_{2 \mathrm{D}}^{\Psi}\left(k_{\perp}, k_{\|}\right) .
\end{gathered}
$$

The 1D omnidirectional spectrum can be obtained by integrating the energy of the Fourier modes over spherical shells delimited by $k$ and $k+d k$,

$$
P_{1 \mathrm{D}}(k)=\sum_{\sqrt{k_{x}^{2}+k_{y}^{2}+k_{z}^{2}}=k} \hat{\Psi}_{3 \mathrm{D}}^{2}\left(k_{x}, k_{y}, k_{z}\right) .
$$

Finally, we define the rms value as

$$
\Psi^{\mathrm{rms}}=\sqrt{\left\langle\boldsymbol{\Psi}^{2}\right\rangle-\langle\boldsymbol{\Psi}\rangle^{2}},
$$

where $\langle\ldots\rangle$ stands for the real-space average over the whole simulation domain.

With these definitions, the initial conditions have $P_{\boldsymbol{B}}^{1 \mathrm{D}} \sim P_{\boldsymbol{u}}^{1 \mathrm{D}} \propto k^{2}$, with $B^{\mathrm{rms}} / B_{0} \sim 0.4$. Since almost all the energy is concentrated at $k d_{\mathrm{i}} \sim 0.25$, the estimated nonlinear time at the beginning of the simulation is $t_{\mathrm{NL}} \sim 10 \Omega_{\mathrm{i}}^{-1}$.

\section{Results}

\subsection{Time Evolution and Development of a Turbulent Cascade}

In Figure 1, we report the time evolution of the rms of the current density, $J^{\text {rms }}$ (black line), of the magnetic field, $B^{\text {rms }}$ (red), and of the ion bulk velocity, $u^{\text {rms }}$ (blue line).

$J^{\text {rms }}$ increases quite rapidly, until a maximum is reached at about $t=t_{\max } \equiv 160 \Omega_{\mathrm{i}}^{-1}$ (marked with a vertical dashed line), and then it declines smoothly and slowly. The maximum corresponds to about $15 t_{\mathrm{NL}}$, which is of the same order as what is observed in previous $2 \mathrm{D}$ simulations using a very similar setup (Franci et al. 2015a). With the peak in $J^{\mathrm{rms}}$ typically being regarded as an indicator of the maximum turbulent activity (Mininni \& Pouquet 2009), the analysis of the spectral properties in Section 3.2 will be performed at $t=t^{\max }$. Moreover, the turbulent activity is observed to be quite stable afterward, so that all the considerations remain valid until the end of the simulation $\left(t=240 \Omega_{\mathrm{i}}^{-1}\right)$. Both $B^{\mathrm{rms}}$ and $u^{\mathrm{rms}}$ decline quasi-steadily all over the simulation, with an excess of magnetic over kinetic energy of about 10\%-15\% maintained throughout the whole evolution.

In Figure 2, we report 3D pseudo-color plots of the current (panels (a)-(c)) and magnetic field structures (panels (d)-(f)) at three different times, i.e., $t=40 \Omega, t=80 \Omega$, and $t=$ $160 \Omega_{\mathrm{i}}^{-1}=t^{\max }$. Initially, we observe the formation of intense current sheets having a quasi-2D shape, with a length in the direction of the mean magnetic field that is of the order of the box size, a slightly smaller width, and a much smaller thickness, of the order of the ion inertial length (panel (a)). Later, the number of current structures increases, and some of them are disrupted, likely because of magnetic reconnection, although clear signatures of such events are not easily identifiable by eye (panel (b)). At the time of maximum turbulent activity, the current structures are much more complex and more uniformly distributed all over the physical domain, still being characterized by an elongated shape along the $z$-direction (panel (c)). Correspondingly, the intensity of the magnetic field is shown in panels (d)-(f), along with magnetic field lines (black lines). Large-scale intense magnetic structures are initially isotropic, while they appear more and more filamentary and twisted at later times, with strong gradients in the perpendicular plane and length scale of a few fractions of the box size in the $z$-direction.

This is also seen in the magnetic field lines, which at early times are strongly perturbated along $\boldsymbol{B}_{0}$ and clustered in the perpendicular plane. Initially, they are modulated by longwavelength fluctuations in all directions. At later times, as the fluctuation amplitude decreases, field lines have approximately the same parallel wavelength and their distribution in the perpendicular plane is disordered, because of the many smallscale structures formed in the $(x, y)$ plane. This indicates that a large spectral anisotropy has been developed from the isotropic initial conditions.

The characteristic length scale of the parallel modulation remains equal to approximately $L_{\text {box }} / 8=16 d_{\mathrm{i}}$ at $t \gtrsim 80$, so we will choose this value as an averaging scale in Section 3.3, when comparing the real-space structures of the present 3D simulation with the ones of a previous $2 \mathrm{D}$ run.

\subsection{Fully Developed Quasi-stationary State}

The spectral properties of the magnetic fluctuations at $t=t^{\max }$ are shown in Figure 3 by the isocontours of their 3D axisymmetric spectrum, $P_{3 \mathrm{D}}$ (Equation (1)). These confirm the qualitative behavior seen in Figure 2, i.e., a strong anisotropy develops, with energy cascading mainly in perpendicular wavenumbers. A white isocontour, corresponding to $k_{\perp} d_{\mathrm{i}} \sim 2.5$, separates the MHD range from the kinetic range (as will be inferred from Figure 4). The different spacing of the isolevels in the perpendicular wavenumber allows for a rough qualitative evaluation of the spectral index, which is flatter in the MHD range than in the kinetic one. In the parallel direction, instead, no clear power-law behavior is seen at scales corresponding to the MHD range, and 
(a)

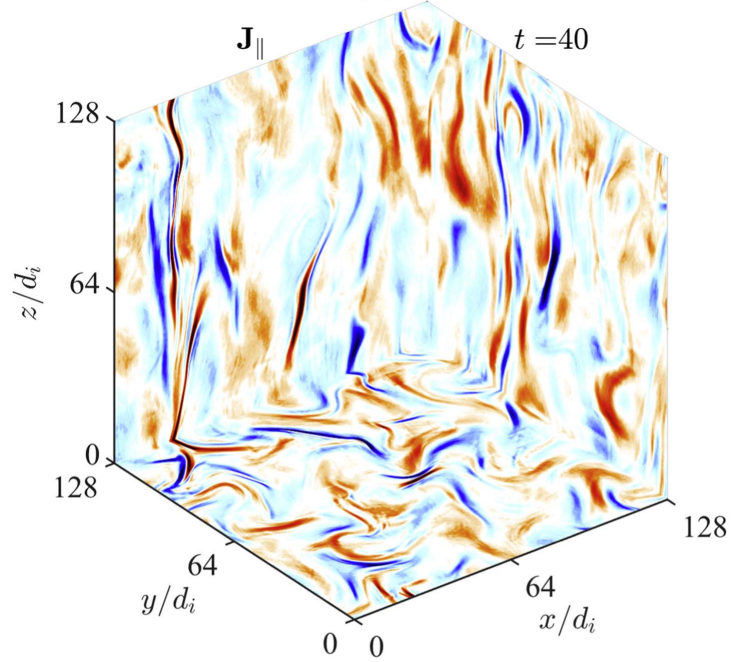

(b)

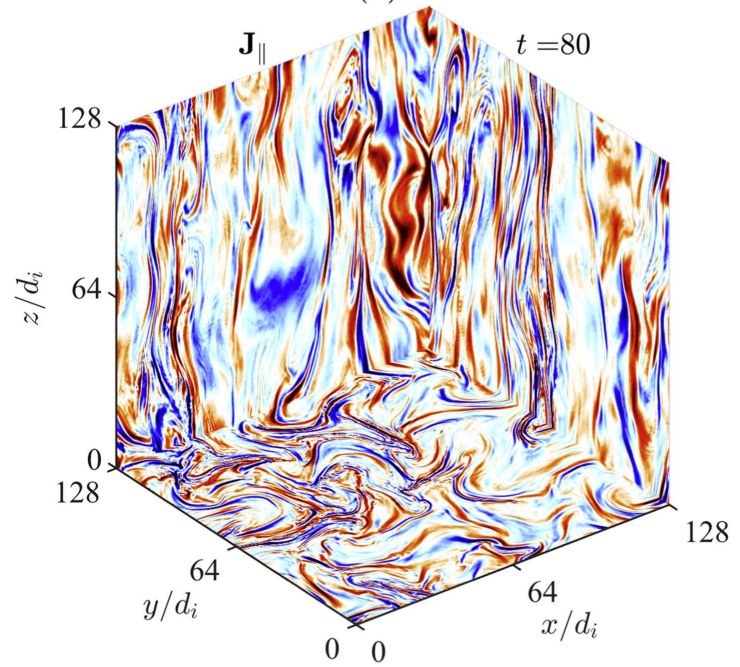

(c)

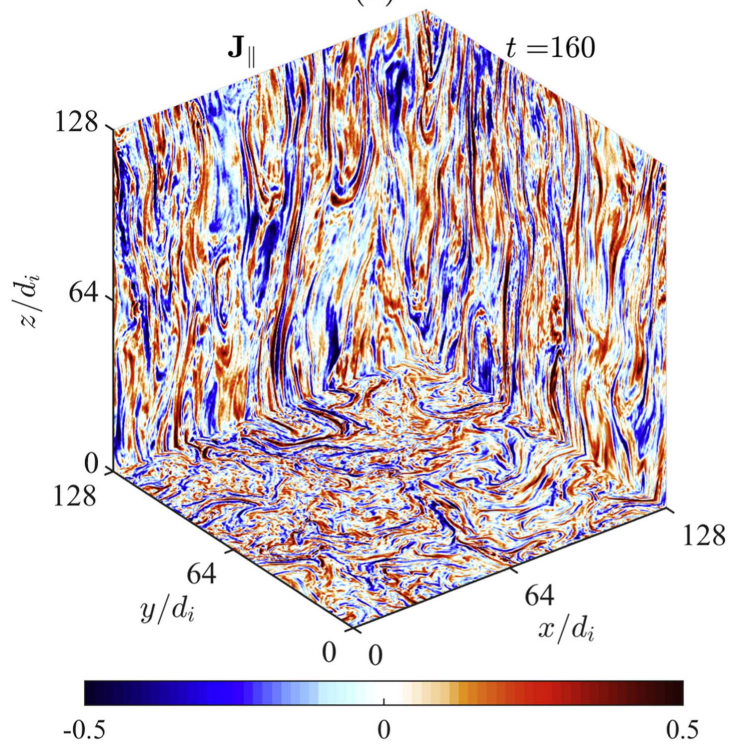

(d)

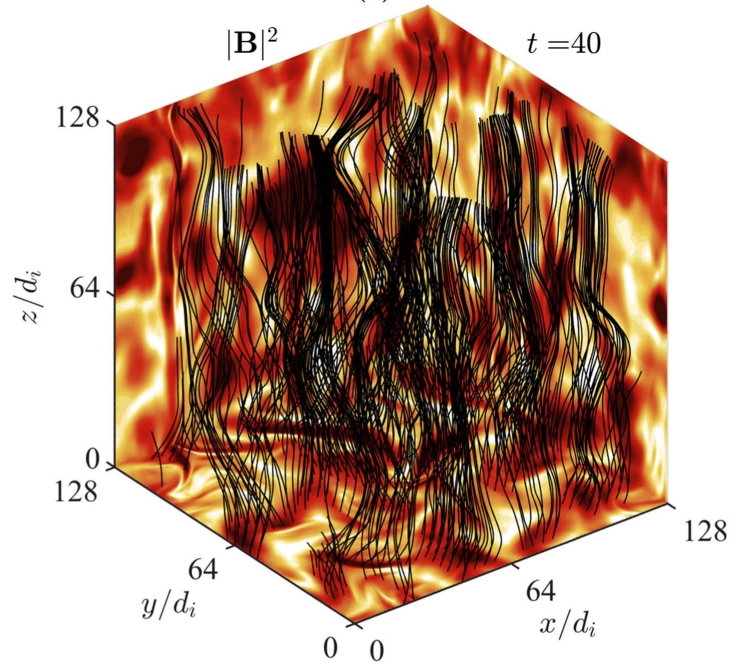

(e)

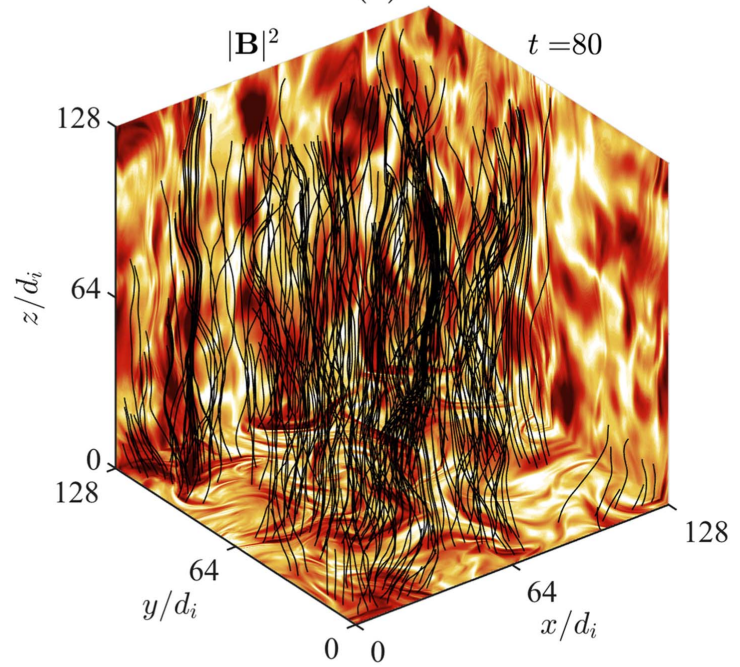

(f)

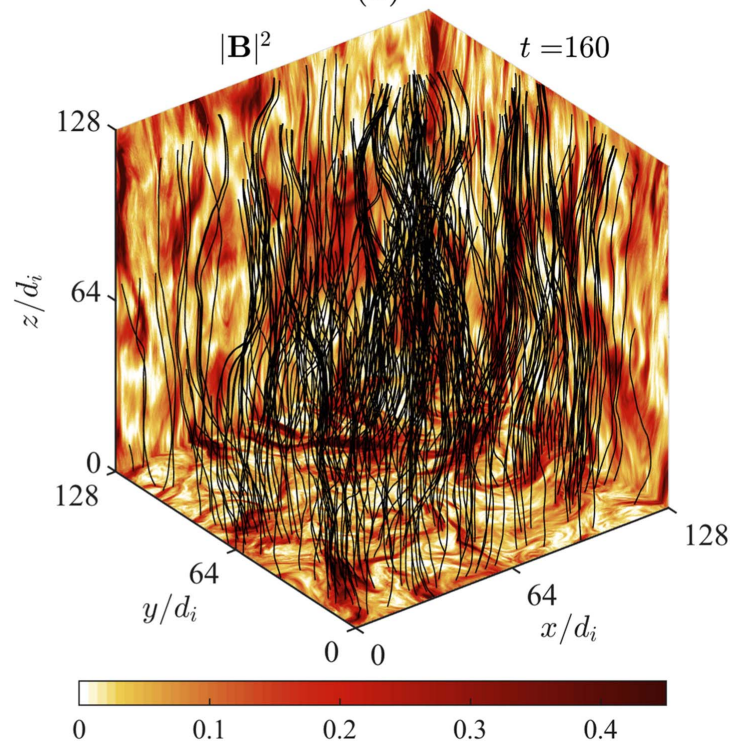

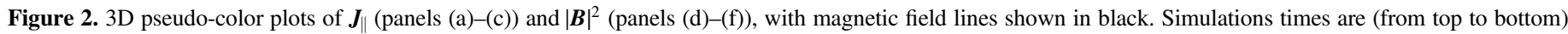
$t=40 \Omega, t=80 \Omega$, and $t=160 \Omega_{\mathrm{i}}^{-1}$. 


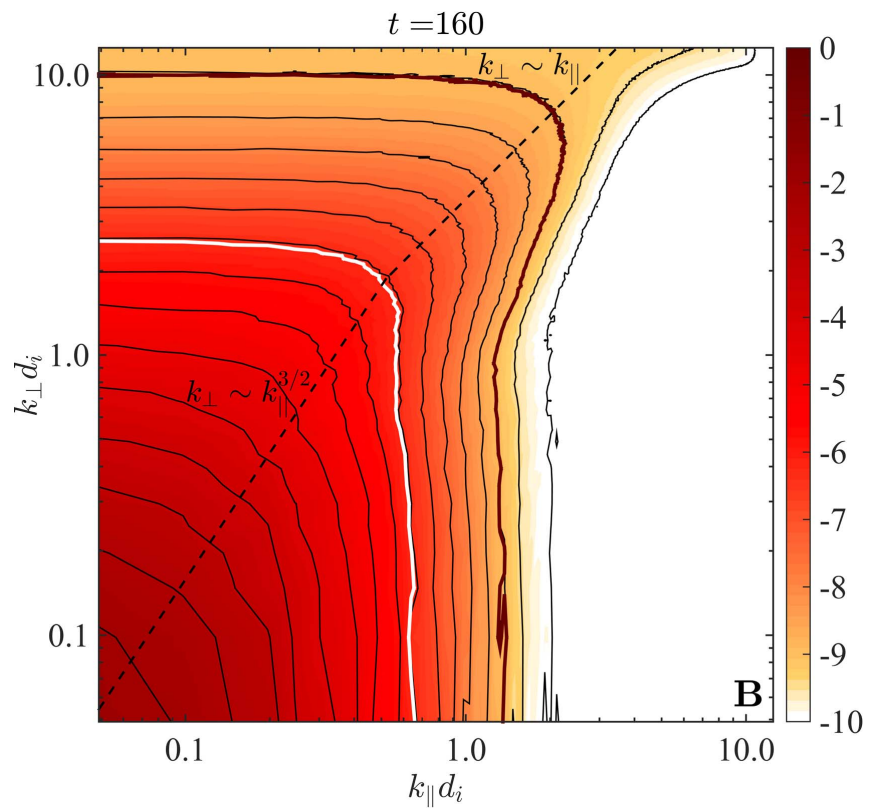

Figure 3. Isocontours of the 3D spectrum of the magnetic fluctuations, $P_{3 \mathrm{D}}^{\boldsymbol{B}}$, in the $\left(k_{\|}, k_{\perp}\right)$ space, at $t=t^{\max }$.

the transition between the two ranges occurs at smaller wavenumbers.

Note that the spectral anisotropy is scale dependent in the MHD range, while it is scale independent in the kinetic range, as suggested by the vertices of the isolevels being aligned approximately with the reference scaling (black dashed lines), $k_{\|} \sim k_{\perp}^{2 / 3}$ and $k_{\|} \sim k_{\perp}$, respectively.

The temporal evolution of the 3D spectrum (not shown) reveals that the cascading energy first reaches the maximum wavenumber (corresponding to the grid size) in the perpendicular direction and then spreads in the parallel direction for high values of $k_{\perp}$. As a result, an unphysical accumulation occurs at $k_{\perp} d_{\mathrm{i}} \gtrsim 10$ for all parallel wavenumbers. When integrating to obtain the $1 \mathrm{D}$ reduced perpendicular and parallel spectra and the 1D omnidirectional spectra, we will therefore remove this unphysical excitation by putting to zero the power corresponding to all the isocontours that extend beyond the dark red line in the $3 \mathrm{D}$ spectrum.

In Figure 4, we show the 1D reduced perpendicular and parallel spectra (Equations (3) and (4), respectively) for the (a) magnetic, (b) ion bulk velocity, (c) electric, and (d) density fluctuations. The reduced spectra of $\boldsymbol{B}$ obtained by directly integrating $P_{2 \mathrm{D}}^{\boldsymbol{B}}$ (Equation (2)) are shown as dotted lines and labeled as "unfiltered." At all scales, the perpendicular transfer of magnetic energy with respect to the mean field is always dominant with respect to the parallel one, reflecting the strong spectral anisotropy already observed in Figure 3. In particular, $P_{1 \mathrm{D}, \perp}^{\boldsymbol{B}}$ exhibits two different power laws spanning almost two decades in wavevector: a spectral index close to $-5 / 3$ at MHD scales, a transition around ion scales, and a steeper slope $\sim-3$ at sub-ion scales (two reference power laws are shown with black dashed lines). The parallel spectrum, $P_{1 \mathrm{D}, \|}^{\boldsymbol{B}}$, is much steeper at small wavevectors and then flattens for $k_{\|} d_{\mathrm{i}} \gtrsim 1$.

As already mentioned when discussing Figure 3 , a significant energy accumulation, due to numerical effects and not sufficiently removed by the explicit resistivity, occurs for large perpendicular wavevectors, and it spreads out at all scales in the parallel direction. As a consequence, noise can

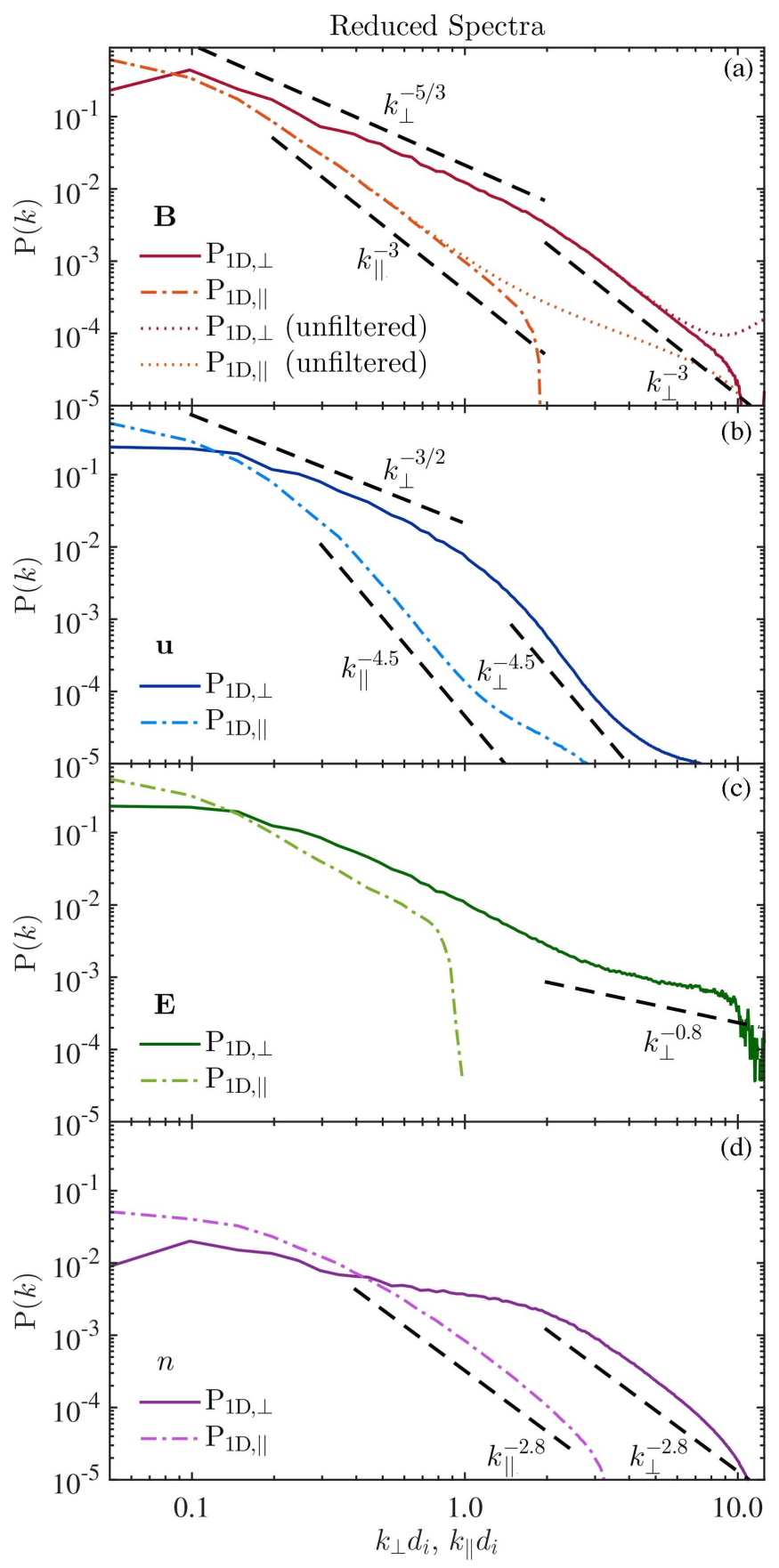

Figure 4. 1D filtered reduced perpendicular and parallel spectra of (a) magnetic, (b) ion bulk velocity, (c) electric, and (d) density fluctuations. Additionally, the unfiltered spectra are shown for the magnetic fluctuations, with dotted lines for comparison. Characteristic power laws are shown with black dashed lines as a reference.

significantly affect $P_{1 \mathrm{D}, \|}^{\boldsymbol{B}}$, causing the flattening observed for $k_{\|} d_{\mathrm{i}} \gtrsim 1$. We estimate a filtering scale, $k_{\text {filter }} d_{\mathrm{i}}$, at which this effect unphysically alters the spectral behavior and we filter out all the isocontours of $P_{2 \mathrm{D}}$ beyond that threshold (shown for the magnetic field as a white line in Figure 3). Specifically, we choose $k_{\text {filter }} d_{\mathrm{i}}=10$, which corresponds to the perpendicular wavevector where $P_{1 \mathrm{D}, \perp}^{\boldsymbol{B}}$ starts to artificially increase. By testing different values for the threshold, we have checked that the filtering procedure does not affect the scales larger than $k_{\text {filter }}$, while it removes artificial features only due to the power accumulation (especially in the parallel spectra). 


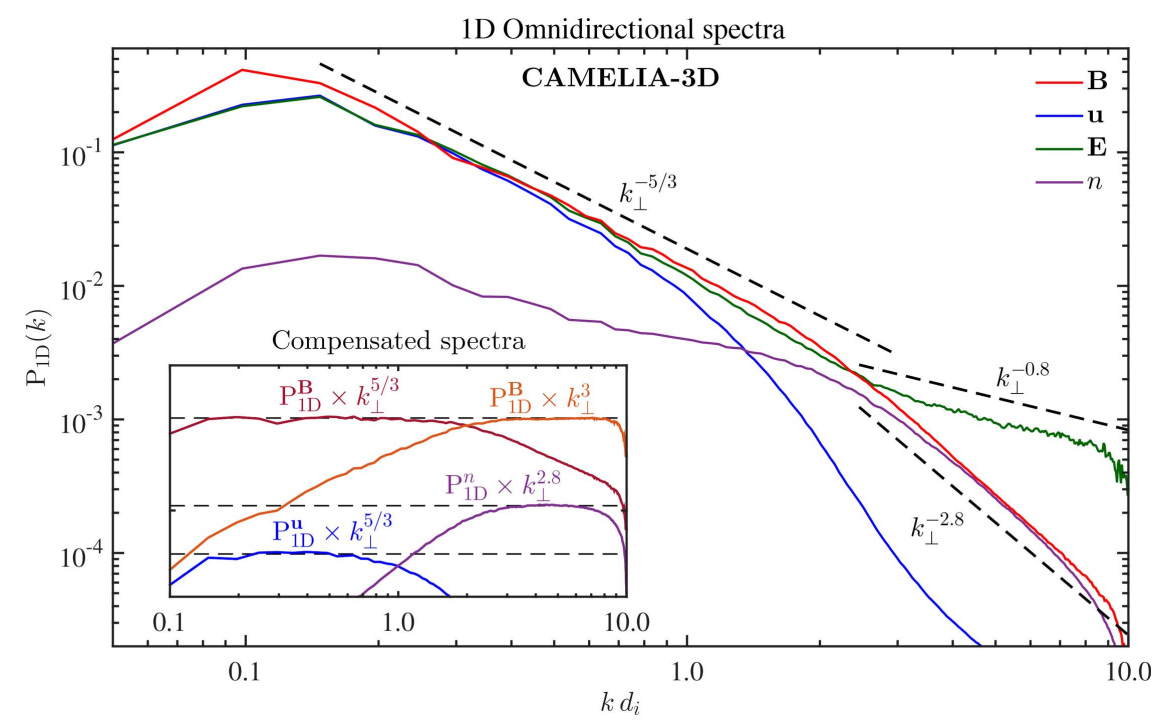

Figure 5. 1D omnidirectional spectra of magnetic (red), ion bulk velocity (blue), electric (green), and density (purple) fluctuations. Characteristic power laws are also shown with dashed black lines as a reference. The inset reports the same spectra compensated by the powers of $k$ indicated with respective colors.

The smallest scales are problematic for the other fields as well, due to the finite number of particles (affecting the density and velocity) and to the computation of finite-difference derivatives of numerically affected fields (for the electric field). We thus repeat the same filtering procedure for all the fields, setting the same filtering scale as for $\boldsymbol{B}$.

The filtered reduced spectra of magnetic fluctuations are shown in Figure 4(a) with solid lines. No flattening is now seen in $P_{1 \mathrm{D}, \|}^{B}$. In the range $0.2 \lesssim k_{\|} d_{\mathrm{i}} \lesssim 2$, it exhibits a power law with a slope very close to that of $P_{1 \mathrm{D}, \perp}^{B}$ for $1 \lesssim k_{\perp} d_{\mathrm{i}} \lesssim 10$.

The reduced perpendicular spectrum of the ion bulk velocity, $P_{1 \mathrm{D}, \perp}^{u}($ Figure 4(b)), shows a sort of power-law-like behavior at large scales. This is less extended than the magnetic field's, so that it is not possible to clearly distinguish whether its spectral index is closer to $-5 / 3$ or $-3 / 2$. At scales corresponding to $k_{\perp} d_{\mathrm{i}} \gtrsim 1, P_{1 \mathrm{D}, \perp}^{u}$ drops very rapidly, reaching the ppc noise level at slightly smaller scales. Again, its parallel counterpart in the interval $k_{\|} d_{\mathrm{i}} \sim[0.3,1]$ resembles the perpendicular spectrum, revealing a power-law shape with a spectral index significantly steeper than the magnetic field's, close to $\sim-4.5$.

The reduced perpendicular spectrum of the electric field, $P_{1 \mathrm{D}, \perp}^{E}$ (Figure 4(c)), follows $P_{1 \mathrm{D}, \perp}^{u}$ at large scales, with $\boldsymbol{u} \times \boldsymbol{B}_{0}$ being the dominant contribution to the generalized Ohm's law (Franci et al. 2015a). It flattens when reaching the ion scales, becoming the most energetic field. Its spectral index is $\sim-0.8$ at sub-ion scales (see the reference dashed line), consistently with the fact that the dominant contributions are the ones coming from the Hall term and from the electron pressure gradient term (Franci et al. 2015a). Note that the electric field is the most affected field by numerical noise at small scales (susceptible to either finite-difference derivative approximations or ppc noise effects); thus, the noise is dominant already at $k_{\|} d_{\mathrm{i}} \gtrsim 1$ in the less powerful $P_{1 \mathrm{D}, \|}^{E}$.

The reduced spectra of the density fluctuations, $P_{1 \mathrm{D}, \perp}^{n}$ and $P_{1 \mathrm{D}, \|}^{n}$, are shown in Figure 4(d). The former is almost flat at intermediate scales, with a slope of the order of $\sim-0.7$, although it seems to be slightly steeper at large scales. A transition is clearly observed around ion scales, followed by a power law with a spectral index of $\sim-2.8$. As for the other fields, $P_{1 \mathrm{D}, \|}^{n}$ exhibits the same profile as its perpendicular counterpart, shifted toward larger $k$-vectors, with the sizable difference that the parallel transfer of energy is not negligible at all scales larger than the injection scales, representing instead the dominant contribution up to $k_{\|} d_{\mathrm{i}} \sim 0.4$.

A comprehensive overview of the 1D omnidirectional filtered power spectra of all fields is provided in Figure 5. Since they all exhibit power-law behavior but with different slopes, we also show them compensated in the inset, in order to allow for a quantitative evaluation of the spectral indices and to better appreciate the agreement with solar wind observations. They are all qualitatively very similar to the corresponding reduced perpendicular spectra, so we won't describe all the details again here. It is just worth noting that due to the integration of the 3D spectrum over shells of constant $k$, the power of fluctuations at small wavevector in $P_{1 \mathrm{D}}$ is slightly larger for all fields. This makes the spectral index of the ion bulk velocity spectrum be closer to $-5 / 3$ (see the inset). Moreover, the density spectrum resembles a sort of triplepower-law behavior, with slopes $\sim-1.0, \sim-0.7$, and $\sim-2.8$ in the ranges $0.2 \lesssim k d_{\mathrm{i}} \lesssim 0.6,0.6 \lesssim k d_{\mathrm{i}} \lesssim 2$, and $2 \lesssim k d_{\mathrm{i}} \lesssim 8$, respectively, which is suggestive of solar wind observations. The three ranges are too small here to firmly infer the presence of three different power laws, and anyway the spectral indices do not correspond to the observed values of $\sim-1.8, \sim-1.1, \sim-2.8$ (Šafránková et al. 2015). However, interestingly, the density spectral behavior in an earlier phase of the evolution (not shown here) is very similar to the one observed in the solar wind. For example, at $t=80$, the three spectral indices are compatible with $\sim-5 / 3, \sim-1.1$, and $\sim-2.8$. Finally, Figure 5 clearly shows how equipartition between magnetic and density fluctuations is achieved at kinetic scales, consistently with observations (Chen et al. 2013). A more quantitative comparison between $P_{1 \mathrm{D}}$ and $P_{1 \mathrm{D}, \perp}$ will be later shown in Figure 7.

\subsection{Comparison with the $2 D$ Case}

In this section, we directly compare the results from the present 3D simulation with those of the 2D HPIC simulation already discussed in Franci et al. (2015a, 2015b). The initial conditions are quite similar, since they are both initialized with 
a mean magnetic field and the same kind of fluctuations. These are linearly polarized Alfvénic fluctuations, which are injected at approximately the same scales, $k d_{\mathrm{i}} \lesssim 0.25$ and $k d_{\mathrm{i}} \lesssim 0.28$, in $3 \mathrm{D}$ and $2 \mathrm{D}$, respectively, and reach a comparable level at the peak of the turbulent activity, $\boldsymbol{B}^{\mathrm{rms}} \sim 0.3$ and $\sim 0.25$. The ion and electron plasma beta are also the same, $\beta_{i}=\beta_{e}=0.5$. The only main difference is the resolution, since the same accuracy is currently not feasible in $3 \mathrm{D}$, due to computational limitations: the 2D simulation has a better spatial resolution $\left(\Delta x=\Delta y=d_{\mathrm{i}} / 8\right)$, a larger extension $\left(256 d_{\mathrm{i}}\right)$, and a higher number of ppc (8000), allowing us to cover a more extended, although similar, range in $k$-vectors, i.e., $k d_{\mathrm{i}}=[0.025,25]$ in $2 \mathrm{D}$ and $[0.05,12.5]$ in $3 \mathrm{D}$.

In Figure 6, we show the magnetic field structures in real space. In the top panel, we report $|\boldsymbol{B}|^{2}$ in a subgrid of the 2D simulation, with the same size of the whole 3D domain. This is compared with a $2 \mathrm{D}$ perpendicular cut of the $3 \mathrm{D}$ box taken at $z=64 d_{\mathrm{i}}$ (middle panel) and with an average of the 3D box along the $z$-direction over a length of $L_{\mathrm{box}} / 8=16 d_{\mathrm{i}}$ (bottom). In the $2 \mathrm{D}$ case, clear close vortices are present, together with more elongated and filamentary structures and small regions of strong gradients with width of the order of $d_{\mathrm{i}}$. Conversely, the 3D case looks quite different when a single perpendicular plane is selected, since circlelike structures are completely absent. The picture changes when averaging along the direction of the mean field, over about a correlation length in the parallel direction (bottom panel). Now structures resembling the shape of vortices emerge, with a comparable size to the 2D case, and the same holds for thin elongated regions with strong gradients, resembling ion-scale current sheets. This suggests that the magnetic structures have the shape of flux tubes oriented in the z-direction, and current structures have the shape of sheets, with a width and thickness of the same order as the 2D case. All of these structures are modulated in the direction of the mean magnetic field, similarly to the magnetic field lines represented in Figure 2.

A direct comparison of the spectral properties between the $3 \mathrm{D}$ and the 2D cases is reported in Figure 7, where we show the spectra of (a) magnetic, (b) velocity, (c) electric, and (d) density fluctuations. For the 3D case, we report both $P_{1 \mathrm{D}}$ (dashed lines) and $P_{1 \mathrm{D}, \perp}$ (dot-dashed). We do not filter the $2 \mathrm{D}$ spectra since the energy accumulation and ppc noise become important at small scales that are not resolved in the 3D run.

The magnetic field spectra are very close to each other, following basically the same power laws both at fluid scales, where the $3 \mathrm{D}$ simulation shows a higher power in agreement with the higher rms, and in the sub-ion range, where they exactly overlap. Moreover, the scale of the transition between the two regimes is unchanged.

The spectra of all the other fields look essentially the same in the kinetic range (except for the higher noise level of the 3D simulation). Some differences are instead observed at larger (fluid) scales, e.g., a different level of fluctuations in the density and in the ion bulk velocity (and, consequently, in the electric field). These could be related to the higher level of magnetic fluctuations injected as initial conditions in 3D, which, for example, produces a higher level of compressible fluctuations at the largest scales, or to a strong Alfvénic coupling between the ion bulk velocity and magnetic fluctuations, parallel to the mean field at $t=0$, which forces the velocity spectrum to be more coupled to the magnetic field's at large scales.

A quantitative analysis of the power spectra of plasma and electromagnetic fluctuations for the $2 \mathrm{D}$ and the $3 \mathrm{D}$ cases is also
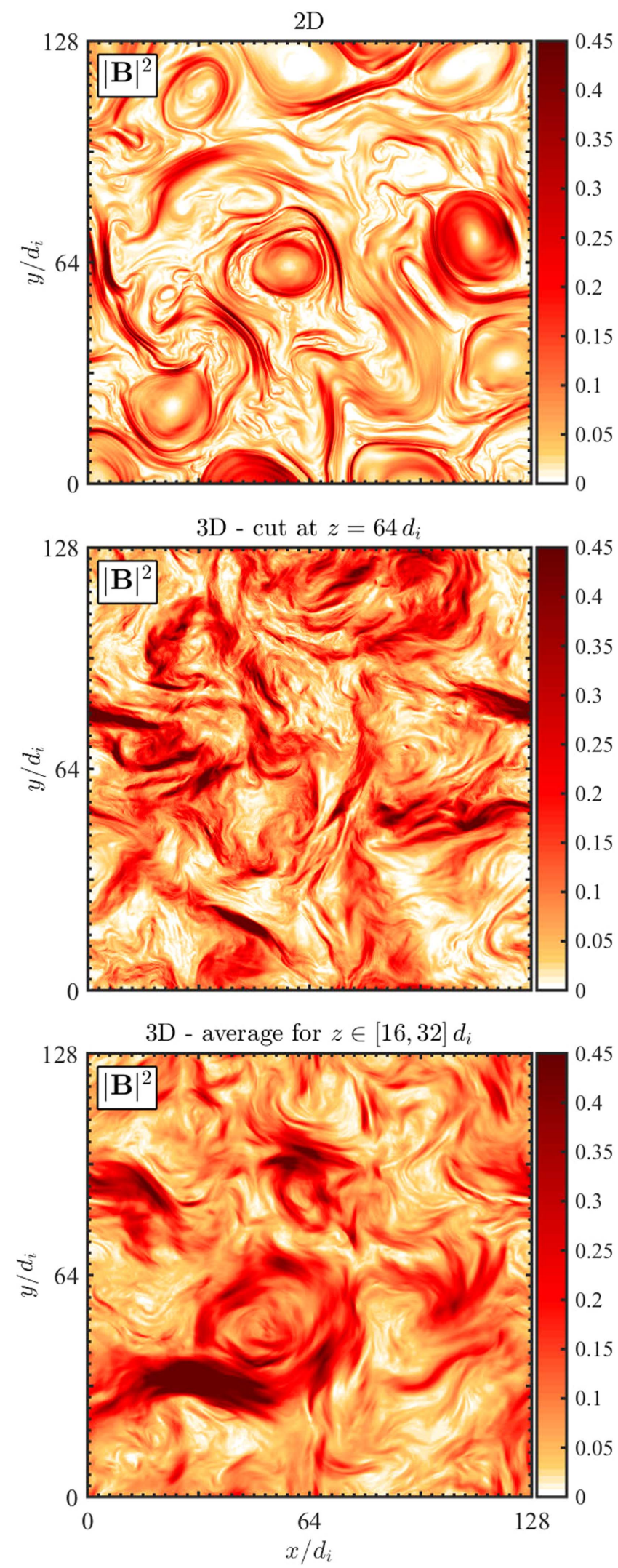

Figure 6. Comparison of the magnetic structures in real space between a previous 2D HPIC simulation (Franci et al. 2015b) and the present 3D one. For the former, we show a subgrid (top panel) with the same dimension of the full 3D box, while for the latter we show both a cut on an $(x, y)$ plane perpendicular to the mean magnetic field (middle panel) and an average along the $z$-direction over a length of the order of $L_{\mathrm{box}} / 8=16 d_{\mathrm{i}}$ (bottom panel). 


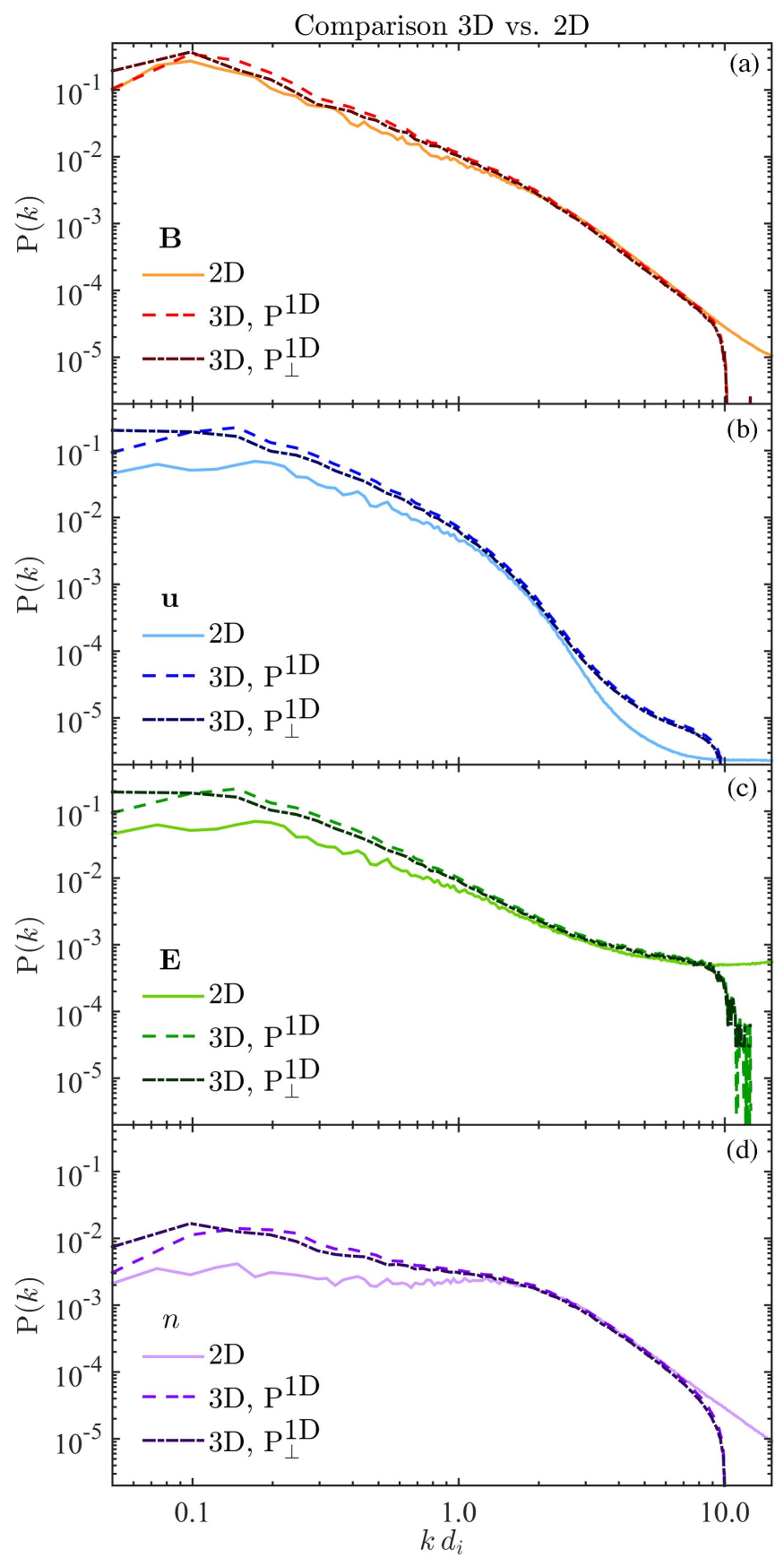

Figure 7. Comparison between the spectral properties of the 2D and the 3D simulations: (a) magnetic, (b) ion bulk velocity, (c) electric, and (d) density fluctuations. For the 3D case, we show both the reduced perpendicular spectra and the $1 \mathrm{D}$ omnidirectional spectra.

shown in Franci et al. (2017a), where the local slopes in small intervals spanning both the fluid and the kinetic scales are directly compared.

In Figure 8, we further compare the three nondimensional ratios already shown for the 2D simulation in Figure 7 of Franci et al. (2015b): the ratio between the perpendicular electric fluctuations (normalized by the Alfvén speed) and the perpendicular magnetic fluctuations,

$$
R_{E_{\perp} B_{\perp}}=\frac{\delta E_{\perp} / v_{A}}{\delta B_{\perp}}
$$

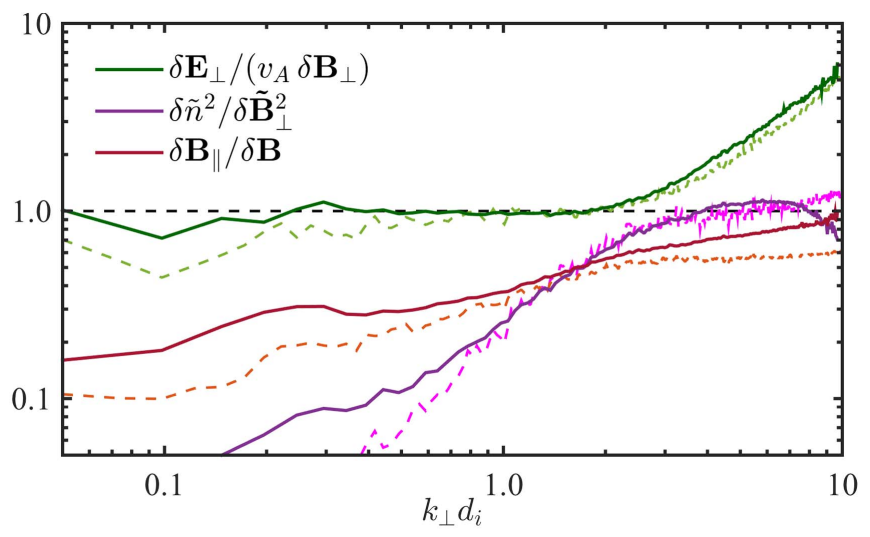

Figure 8. Comparison between the spectral properties of the $2 \mathrm{D}$ and the $3 \mathrm{D}$ simulations: ratio of the perpendicular electric field to the perpendicular magnetic field (green), ratio of the normalized density to the perpendicular magnetic fluctuations (purple), and magnetic compressibility (red).

the magnetic compressibility,

$$
R_{B_{\|} B}=\frac{\delta B_{\|}}{\delta B},
$$

and the ratio between the normalized energy of density and perpendicular magnetic fluctuations,

$$
R_{n B_{\perp}}=\frac{\delta \tilde{n}^{2}}{\delta \tilde{B}_{\perp}^{2}}=\frac{\sqrt{\Gamma} \delta n / n_{o}}{\delta B_{\perp} / B_{0}}
$$

where $\Gamma=3 / 4$ for $\beta_{i}=0.5$ and $T_{e}=T_{i}$, as in the present case (Schekochihin et al. 2009; Boldyrev et al. 2013). The ratios for the $3 \mathrm{D}$ case are shown with solid lines, while the $2 \mathrm{D}$ case is represented by lighter dashed lines.

The ratio of electric to magnetic field (green) shows three different regions. At the injection scales, $k d_{\mathrm{i}} \lesssim 0.25$, it is smaller than 1 , the electric field being coupled to the velocity field via the MHD term in the generalized Ohm's law (see discussion of Figure 4). The MHD range value of $R_{E_{\perp} B_{\perp}}$ is larger in 3D with respect to 2D because of the stronger coupling between the velocity and the magnetic fluctuations (the residual energy, i.e., the excess of magnetic energy over kinetic energy, is smaller, and concurrently the spectral index of the velocity spectrum is closer to $-5 / 3$, like the magnetic field's, rather than to $-3 / 2$, as in $2 \mathrm{D}$ ). At intermediate scales, before the transition to the kinetic regime $\left(0.25 \lesssim k d_{\mathrm{i}} \lesssim 2\right)$, $R_{E_{\perp} B_{\perp}}$ is exactly 1 , the electric field being coupled to the magnetic fluctuations. As seen in Figure 7(a), the magnetic field spectra are almost indistinguishable in the two simulations, meaning that the coupling is more effective in $3 \mathrm{D}$ than in 2D. At wavenumbers in the kinetic range $\left(k d_{\mathrm{i}} \gtrsim 2\right)$ the two simulations have a negligible difference and $R_{E_{1} B_{\perp}}$ increases with the same (linear) scaling, as a direct consequence of Ohm's law, and consistently with observed frequency spectra in the solar wind frame (Bale et al. 2005; Matteini et al. 2017).

The magnetic compressibility (red) is quite small in the MHD range and increases toward smaller and smaller scales (Lacombe et al. 2017). Thus, at the injection scales, the magnetic fluctuations are mainly perpendicular to the mean field, as imposed by our initial conditions, but they tend to become more isotropic at ion and sub-ion scales. The qualitative trend of $R_{B_{\mid B}}$ from fluid to kinetic scales is similar with respect to the $2 \mathrm{D}$ case. However, its value is slightly larger 
in $3 \mathrm{D}$ in the whole range of scales, of a factor of $\sim 1.5$, which is compatible with the different initial level of fluctuations $\left(B^{\mathrm{rms}}=0.38\right.$ versus 0.24$)$. Note that in $2 \mathrm{D}$ the ratio $R_{B_{\|} B}$ reaches a plateau for $k d_{\mathrm{i}} \gtrsim 2$, corresponding to component isotropy, while in the $3 \mathrm{D}$ case it increases all the way down to the smallest scales. We have checked that this is not an effect of the filtering procedure.

Finally, the ratio between the density and the magnetic fluctuations (purple) is very small at MHD scales, although larger in 3D than in 2D. In this range, the density spectrum in $3 \mathrm{D}$ is dominated by parallel wavevectors, and it is more energetic than in the 2D case (compare Figure 4(d) and Figure $7(d)$ ). The difference could thus be attributed to the parallel scales becoming accessible only in $3 \mathrm{D}$. For $k d_{\mathrm{i}} \gtrsim 1$, $R_{n B_{\perp}}$ is almost the same in the two cases, with the $3 \mathrm{D}$ one showing a decrease around $k d_{\mathrm{i}} \sim 8$, only due to the filtering procedure. A ratio of 0.75 is observed on average in solar wind data (Chen et al. 2013). In both our 2D and 3D simulations an approximate plateau at a value of $\sim 1$ is found, indicating that even in 3D the perpendicular fluctuations can be reasonably described by kinetic Alfvén fluctuations in an intermediatebeta case.

\section{Discussion and Conclusions}

We have presented results from a large-size hybrid-kinetic 3D simulation of freely decaying turbulence in the presence of a mean magnetic field. The high resolution adopted in terms of both number of grid points $\left(512^{3}\right)$ and ppc (2048) allows the simulation to develop a turbulent cascade spanning both the MHD and the kinetic ranges of scales. As a consequence, we obtain remarkably stable and well-defined power spectra of the magnetic, ion bulk velocity, electric, and density fluctuations, covering two full decades in $k_{\perp}$ and slightly more than a full decade in $k_{\|}$. A convergence study on the effects of the number of grid points and of ppc, in support of the present results, is provided in Franci et al. (2017a).

The main results of the present work are that (i) the turbulent cascade mainly develops in the direction perpendicular to the mean magnetic field, so that a strong spectral anisotropy is achieved, despite the isotropic initial conditions; (ii) the 1D omnidirectional spectra (as well as the 1D reduced perpendicular spectra) of all fields exhibit power-law behavior spanning both the MHD and the kinetic range, with spectral indices that are in remarkable agreement with solar wind observations; and (iii) the comparison between the present 3D simulation and a previous 2D simulation with similar parameters shows that the spectral behavior of all fields near and below the ion characteristic scales is not affected by a reduced $2 \mathrm{D}$ geometry.

The 3D spectrum of the magnetic fluctuations shows that turbulence develops a strong anisotropy, with more power in the perpendicular wavevectors. In particular, the anisotropy is observed to be scale dependent at large MHD scales. On the contrary, it becomes scale independent at small kinetic scales. This last result is also confirmed by the 1D reduced parallel and perpendicular spectra: for all the fields, the former exhibits the same power law of the latter, just shifted toward larger scales. At even larger scales, the parallel spectra do not show any power-law behavior, possibly because the physical box size along $B_{0}$ is too short to accommodate the long parallel wavelengths expected in a strong-turbulence regime. We expect anisotropy to change when analyzed in a reference frame attached to the local mean field. A more quantitative analysis and an articulated discussion about the spectral anisotropy are beyond the scope of this paper and will be the subject of future work.

The large physical extent in the perpendicular directions allows us to recover power-law scaling in both the MHD and the kinetic range, covering simultaneously two full decades in wavenumbers and the transition between the two regimes. The 1D omnidirectional spectra recover several spectral indices found in larger 2D HPIC simulations with similar initial conditions and plasma parameters (Franci et al. 2015a, 2015b).

In particular, the magnetic field spectrum has the same properties as in 2D HPIC simulations at both MHD and kinetic scales: a double-power-law behavior with a Kolmogorov-like index of $-5 / 3$ at low $k$ values, followed by a steeper spectrum with index $\sim-3$ in the kinetic range. The transition (break) occurs around ion scales, $k d_{\mathrm{i}} \sim 2$, just as in the 2D case with the same ion plasma beta. This support our previous 2D numerical study, in which we provided numerical evidence that the plasma beta controls the position of the break (Franci et al. 2016).

The ion bulk velocity, electric field, and density spectra are again similar to the corresponding 2D ones, but only at kinetic scales. The ion bulk velocity spectrum drops dramatically, with a trend that can be approximated as a power law with a steep slope of $\sim-4.5$. The electric field spectrum flattens with a power-law index of $\sim-0.8$, becoming the dominating type of fluctuations. The density spectrum steepens and reaches a sort of equipartition with the magnetic energy at sub-ion scales. Its power-law index, $\sim-2.8$, is close but not identical to that of the magnetic field spectrum. All these spectral properties in agreement with the $2 \mathrm{D}$ case indicate that, because of the large anisotropy, the coupling between the electric field fluctuations, density fluctuations, and the parallel component of magnetic fluctuations found in 2D at kinetic scales (Franci et al. 2015b) continues to hold in the full 3D geometry. Note that all the above spectral indices are also in agreement with solar wind observations (see also Figure 1 of Franci et al. 2017a).

At large scales, on the contrary, the velocity, electric field, and density spectra have noticeable differences with respect to the $2 \mathrm{D}$ case and to observations in the solar wind.

The velocity fluctuations have a power-law index closer to $-5 / 3$ than to $-3 / 2$, as found instead in $2 \mathrm{D}$ simulations and in observations. In our $3 \mathrm{D}$ run, they look strongly coupled to the magnetic fluctuations. Note that the indices $-3 / 2$ and $-5 / 3$ are found for the velocity and magnetic fluctuations in $2 \mathrm{D}$ HPIC simulations with an out-of-plane mean field (Franci et al. 2015a, 2015b) and in weakly compressible 3D MHD simulations with no mean field (Grappin et al. 2016). This suggests that only a weak Alfvénic coupling is achieved in the solar wind, possibly because of the value $B^{\mathrm{rms}} / B_{0} \sim 1$ at hour scales and of the small, but non-negligible, compressibility of velocity fluctuations.

The electric field is strongly coupled to the velocity spectrum at large scales, consistently with the generalized Ohm's law (Franci et al. 2015a) and solar wind observations. Moreover, it keeps following the magnetic field spectrum as the velocity fluctuations start dropping just above the ion scales.

The density spectrum is more energetic and steeper than in the $2 \mathrm{D}$ case at large scales. This could be due to the fact that the energy in parallel wavevectors is larger than in the perpendicular ones, and that parallel scales are clearly absent in 2D. 
Here, instead, this allows for a larger compressibility in the MHD range. Moreover, the higher level of $B^{\text {rms }}$ with respect to the 2D case activates a sufficient power in the density fluctuations, so that the hint of a short decrease (possibly a cascade) is observed at MHD scales. This is consistent with the behavior observed in 2D simulations with larger $B^{\text {rms }}$ (not shown here). Note that the density spectrum resembles a peculiar triple power-law behavior. This is much more evident and clear when the turbulent cascade is still developing, since the three observed spectral indices are reproduced quite well, rather than when a fully developed quasi-stationary state has been achieved.

According to the spectral anisotropy, large-scale structures and currents in real space are preferentially aligned to the mean magnetic field, with gradients being more developed in the perpendicular plane. When averaging over a parallel correlation length, such structures are isotropic in the perpendicular plane and acquire the vortex-like shape characteristic of 2D runs. In a perpendicular cut, small-scale currents have roughly the same thickness and width found in the 2D geometry. Their aspect ratio is approximately conserved after averaging in the parallel direction, indicating that currents are sheet-like structures with a long-wavelength parallel modulation and a weak twist.

The agreement between the $2 \mathrm{D}$ and the $3 \mathrm{D}$ cases is important from both a pratical and a physical point of view. On the one hand, it validates the use of $2 \mathrm{D}$ simulations for all those cases where the study of spectral properties is involved and the use of a large collection of simulations with different values of parameters is required, e.g., for convergence studies (Franci et al. 2015a), or for parameter studies (Franci et al. 2016). On the other hand, it suggests that the dominant process(es) responsible for the ion-scale spectral break in the magnetic field and for the kinetic-scale turbulent cascade are likely not inhibited in a reduced 2D geometry. In particular, the fact that the scale of the break is unmoved passing from the 2D evolution to $3 \mathrm{D}$ when the same ion plasma beta is set supports our previous finding that beta is likely the only main parameter controlling such a scale. Moreover, the fact that the width of the $2 \mathrm{D}$ current sheets in the $3 \mathrm{D}$ simulation seems to be of the same order of the 1D current sheets in the 2D simulation (see the bottom panel of Figure 6) might support, although only qualitatively at this level, the idea that the disruption of current structures via magnetic reconnection may be the main factor responsible for the break and the onset of the sub-ion-scale cascade even in more realistic 3D turbulence. Although the complex shape of magnetic and current structures makes the identification of reconnection sites in 3D much more difficult than in 2D, a preliminary analysis (not presented here) shows a correspondence between the first peak in the maximum of the current and the development of the kinetic power law, in agreement with the findings of Franci et al. (2017b) in 2D. A more quantitative analysis of the current structures is necessary in order to confirm this scenario.

As a final remark, we note that the good agreement between the $2 \mathrm{D}$ and the $3 \mathrm{D}$ numerical results may find theoretical support and a possible interpretation in Zank et al. (2017). There, the nearly incompressible magnetohydrodynamic turbulence in the intermediate- and small-beta regimes is shown to comprise the presence of majority $2 \mathrm{D}$ and minority slab fluctuations. As a consequence, the 3D power spectrum, being the superposition of those two components, clearly exhibits an anisotropic behavior. A comparison with theoretical predictions, also for cases with different values of the plasma beta, will be the subject of future work.

The authors wish to acknowledge valuable discussions with E. Papini, C. H. K. Chen., S. S. Cerri, F. Califano, D. Burgess, and O. Alexandrova. L.F. is funded by Fondazione Cassa di Risparmio di Firenze through the project "Giovani Ricercatori Protagonisti.” P.H. acknowledges GACR grant 15-10057S. We acknowledge PRACE for awarding us access to resource Cartesius based in the Netherlands at SURFsara through the DECI-13 (Distributed European Computing Initiative) call (project HybTurb3D), and the CINECA award under the ISCRA initiative, for the availability of high-performance computing resources and support (grants HP10C877C4 and HP10BUUOJM). Data deposit was provided by the EUDAT infrastructure (https://www.eudat.eu/).

\section{ORCID iDs}

Luca Franci (i) https://orcid.org/0000-0002-7419-0527

Simone Landi (i) https://orcid.org/0000-0002-1322-8712

Andrea Verdini (iD https://orcid.org/0000-0003-4380-4837

Lorenzo Matteini (i) https://orcid.org/0000-0002-6276-7771

Petr Hellinger (iD https://orcid.org/0000-0002-5608-0834

\section{References}

Alexandrova, O., Lacombe, C., Mangeney, A., Grappin, R., \& Maksimovic, M. 2012, ApJ, 760, 121

Alexandrova, O., Saur, J., Lacombe, C., et al. 2009, PhRvL, 103, 165003

Bale, S. D., Kellogg, P. J., Mozer, F. S., Horbury, T. S., \& Reme, H. 2005, PhRvL, 94, 215002

Boldyrev, S., Horaites, K., Xia, Q., \& Perez, J. C. 2013, ApJ, 777, 41

Bruno, R., \& Trenchi, L. 2014, ApJL, 787, L24

Camporeale, E., \& Burgess, D. 2011, ApJ, 730, 114

Cerri, S. S., \& Califano, F. 2017, NJPh, 19, 025007

Cerri, S. S., Califano, F., Jenko, F., Told, D., \& Rincon, F. 2016, ApJL, 822, L12

Cerri, S. S., Servidio, S., \& Califano, F. 2017, ApJL, 846, L18

Chandran, B. D. G., Quataert, E., Howes, G. G., Xia, Q., \& Pongkitiwanichakul, P. 2009, ApJ, 707, 1668

Chang, O., Gary, P. S., \& Wang, J. 2011, GeoRL, 38, L22102

Chen, C. H. K. 2016, JP1Ph, 82, 535820602

Chen, C. H. K., Bale, S. D., Salem, C., \& Mozer, F. S. 2011, ApJL, 737, L41

Chen, C. H. K., Boldyrev, S., Xia, Q., \& Perez, J. C. 2013, PhRvL, 110, 225002

Chen, C. H. K., Leung, L., Boldyrev, S., Maruca, B. A., \& Bale, S. D. 2014, GeoRL, 41, 8081

Chen, C. H. K., Salem, C. S., Bonnell, J. W., Mozer, F. S., \& Bale, S. D. 2012 , PhRvL, 109, 035001

Dong, Y., Verdini, A., \& Grappin, R. 2014, ApJ, 793, 118

Franci, L., Cerri, S. S., Califano, F., et al. 2017b, ApJL, 850, L16

Franci, L., Hellinger, P., Guarrasi, M., et al. 2017a, arXiv:1712.03930

Franci, L., Landi, S., Matteini, L., Verdini, A., \& Hellinger, P. 2015a, ApJ, 812,21

Franci, L., Landi, S., Matteini, L., Verdini, A., \& Hellinger, P. 2016, ApJ, 833,91

Franci, L., Verdini, A., Matteini, L., Landi, S., \& Hellinger, P. 2015b, ApJL, 804, L39

Gary, S. P., Chang, O., \& Wang, J. 2012, ApJ, 755, 142

Gary, S. P., Saito, S., \& Li, H. 2008, GeoRL, 35, 2104

Gómez, D., Martín, L. N., \& Dmitruk, P. 2013, AdSpR, 51, 1916

Grappin, R., Müller, W.-C., \& Verdini, A. 2016, A\&A, 589, A131

Hellinger, P., Landi, S., Matteini, L., Verdini, A., \& Franci, L. 2017a, ApJ, 838,158

Hellinger, P., Matteini, L., Landi, S., et al. 2015, ApJL, 811, L32

Hellinger, P., Verdini, A., Landi, S., Franci, L., \& Matteini, L. 2017b, ApJL, submitted

Howes, G. G., Tenbarge, J. M., Dorland, W., et al. 2011, PhRvL, 107, 035004

Kiyani, K. H., Chapman, S. C., Sahraoui, F., et al. 2013, ApJ, 763, 10

Lacombe, C., Alexandrova, O., \& Matteini, L. 2017, ApJ, 848, 45 
Leamon, R. J., Smith, C. W., Ness, N. F., Matthaeus, W. H., \& Wong, H. K. 1998, JGR, 103, 4775

Loureiro, N. F., \& Boldyrev, S. 2017, ApJ, 850, 182

MacBride, B. T., Forman, M. A., \& Smith, C. W. 2005, in ESA Special Publication 592, Solar Wind 11/SOHO 16, Connecting Sun and Heliosphere, ed. B. Fleck, T. H. Zurbuchen, \& H. Lacoste (Paris: ESA), 613 MacBride, B. T., Smith, C. W., \& Forman, M. A. 2008, ApJ, 679, 1644

Mallet, A., Schekochihin, A. A., \& Chandran, B. D. G. 2017, MNRAS, 468, 4862

Marino, R., Sorriso-Valvo, L., Carbone, V., et al. 2008, ApJL, 677, L71

Markovskii, S. A., \& Vasquez, B. J. 2010, PhPl, 17, 112902

Matteini, L., Alexandrova, O., Chen, C. H. K., \& Lacombe, C. 2017, MNRAS, 466, 945

Matthews, A. P. 1994, JCoPh, 112, 102

Meyrand, R., \& Galtier, S. 2013, PhRvL, 111, 264501

Mininni, P. D., \& Pouquet, A. 2009, PhRvE, 80, 025401

Parashar, T. N., Shay, M. A., Cassak, P. A., \& Matthaeus, W. H. 2009, PhP1, 16,032310

Passot, T., Henri, P., Laveder, D., \& Sulem, P.-L. 2014, EPJD, 68, 207

Podesta, J. J., Roberts, D. A., \& Goldstein, M. L. 2007, ApJ, 664, 543

Podesta, J. J., \& TenBarge, J. M. 2012, JGR, 117, A10106

Roberts, D. A. 2010, JGRA, 115, 12101

Rodriguez Imazio, P., Martin, L. N., Dmitruk, P., \& Mininni, P. D. 2013, PhPl, 20,052506

Šafránková, J., Němeček, Z., Němec, F., et al. 2015, ApJ, 803, 107

Šafránková, J., Němeček, Z., Němec, F., et al. 2016, ApJ, 825, 121

Sahraoui, F., Goldstein, M. L., Belmont, G., Canu, P., \& Rezeau, L. 2010, PhRvL, 105, 131101

Sahraoui, F., Goldstein, M. L., Robert, P., \& Khotyaintsev, Y. V. 2009 PhRvL, 102, 231102
Sahraoui, F., Huang, S. Y., Belmont, G., et al. 2013, ApJ, 777, 15

Salem, C., Mangeney, A., Bale, S. D., \& Veltri, P. 2009, ApJ, 702, 537

Salem, C. S., Howes, G. G., Sundkvist, D., et al. 2012, ApJL, 745, L9

Schekochihin, A. A., Cowley, S. C., Dorland, W., et al. 2009, ApJS, 182, 310

Servidio, S., Valentini, F., Califano, F., \& Veltri, P. 2012, PhRvL, 108, 045001

Servidio, S., Valentini, F., Perrone, D., et al. 2015, JPIPh, 81, 325810107

Shaikh, D., \& Shukla, P. K. 2009, PhRvL, 102, 045004

Smith, C. W., Hamilton, K., Vasquez, B. J., \& Leamon, R. J. 2006, ApJL, 645, L85

Sorriso-Valvo, L., Marino, R., Carbone, V., et al. 2007, PhRvL, 99, 115001

Stawarz, J. E., Eriksson, S., Wilder, F. D., et al. 2016, JGRA, 121, 11

Stawarz, J. E., Smith, C. W., Vasquez, B. J., Forman, M. A., \& MacBride, B. T. 2009, ApJ, 697, 1119

TenBarge, J. M., \& Howes, G. G. 2013, ApJL, 771, L27

Tessein, J. A., Smith, C. W., MacBride, B. T., et al. 2009, ApJ, 692, 684

Valentini, F., Vásconez, C. L., Pezzi, O., et al. 2017, A\&A, 599, A8

Vasquez, B. J. 2015, ApJ, 806, 33

Vasquez, B. J., Smith, C. W., Hamilton, K., MacBride, B. T., \& Leamon, R. J. 2007, JGRA, 112, A07101

Verdini, A., \& Grappin, R. 2015, ApJL, 808, L34

Verdini, A., \& Grappin, R. 2016, ApJ, 831, 179

Wan, M., Matthaeus, W. H., Karimabadi, H., et al. 2012, PhRvL, 109, 195001

Wan, M., Matthaeus, W. H., Roytershteyn, V., et al. 2015, PhRvL, 114, 175002

Wan, M., Matthaeus, W. H., Roytershteyn, V., et al. 2016, PhPl, 23 042307

Wicks, R. T., Horbury, T. S., Chen, C. H. K., \& Schekochihin, A. A. 2011, PhRvL, 106, 045001

Zank, G. P., Adhikari, L., Hunana, P., et al. 2017, ApJ, 835, 147 